\title{
Complete isotopic distributions of fragments produced in transfer- and fusion-induced reactions
}

O. Delaune ${ }^{1, \mathrm{a}}$, M. Caamaño ${ }^{1,2}$, F. Farget ${ }^{1, \mathrm{~b}}$, O.B. $\operatorname{Tarasov}^{3}$, X. Derkx ${ }^{1}$, K.-H. Schmidt ${ }^{1}$, L. Audouin ${ }^{4}$, A.M. Amthor ${ }^{1}$, C.-O. Bacri ${ }^{4}$, G. Barreau ${ }^{5}$, B. Bastin ${ }^{1}$, D. Bazin ${ }^{3}$, J. Benlliure ${ }^{2}$, B. Blank ${ }^{5}$, L. Cacères ${ }^{1}$, E. Casarejos ${ }^{6}$, B. Fernández-Domínguez ${ }^{2}$, L. Gaudefroy ${ }^{7}$, C. Golabek ${ }^{1}$, S. Grévy ${ }^{1}$, B. Jurado ${ }^{5}$, O. Kamalou ${ }^{1}$, A. Lemasson ${ }^{1}$, S.M. Lukyanov ${ }^{8}$, W. Mittig ${ }^{3,9}$, D.J. Morrissey ${ }^{3,10}$, A. Navin ${ }^{1}$, J. Pereira ${ }^{3}$, L. Perrot ${ }^{4}$, M. Rejmund ${ }^{1}$, T. Roger ${ }^{1}$, M.G. Saint-Laurent ${ }^{1}$, H. Savajols ${ }^{1}$, C. Schmitt ${ }^{1}$, B.M. Sherrill ${ }^{3}$, C. Stodel ${ }^{1}$, J.C. Thomas ${ }^{1}$, and A.C.C. Villari ${ }^{11}$

${ }^{1}$ GANIL, CEA/DSM-CNRS/IN2P3, BP. 55027, 14076 Caen Cedex 5, France

${ }^{2}$ Univ. de Santiago de Compostela, 15706 Santiago de Compostela, Spain

${ }^{3}$ NSCL, Michigan State University, East Lansing, MI 48824, USA

${ }^{4}$ Institut de Physique Nucléaire, CNRS/IN2P3, 91406 Orsay, France

${ }^{5}$ CENBG, UMR 5797 CNRS/IN2P3, Université Bordeaux 1, 33175 Gradignan, France

${ }^{6}$ University of Vigo, 36310, Spain

${ }^{7}$ CEA, DAM, DIF, 91297 Arpajon, France

${ }^{8}$ FLNR, JINR, 141980 Dubna, Moscow region, Russian Federation

${ }^{9}$ Dep. of Physics and Astronomy, Michigan State University, East Lansing, MI 48824, USA

${ }^{10}$ Dep. of Chemistry, Michigan State University, East Lansing, MI 48824, USA

${ }^{11}$ PANTECHNIK S.A., 13, rue de la Résistance, 14400 Bayeux, France

\begin{abstract}
Two fission experiments have been performed at GANIL using ${ }^{238} \mathrm{U}$ beams at different energies and light targets. Different fissioning systems were produced with centre of mass energies from 10 to $240 \mathrm{MeV}$ and their decay by fission was investigated with GANIL spectrometers. Fission-fragment isotopic distributions have been obtained. The evolution with impinging energy of their properties, the neutron excess and the width of the neutron-number distributions, gives important insights into the dynamics of the fusionfission mechanism.
\end{abstract}

\section{Introduction}

The binding energy of the nucleus can be estimated in the framework of the liquid-drop model including shell effects and pairing. Produced in heavy ions collision, the compound nucleus has a certain excitation energy. Assessing the evolution of potential energy with the deformation of a fissioning nucleus,

\footnotetext{
ae-mail: olivier.delaune@cea.fr

be-mail: farget@ganil.fr
}

This is an Open Access article distributed under the terms of the Creative Commons Attribution License 2.0, which permits unrestricted use, distribution, and reproduction in any medium, provided the original work is properly cited. 

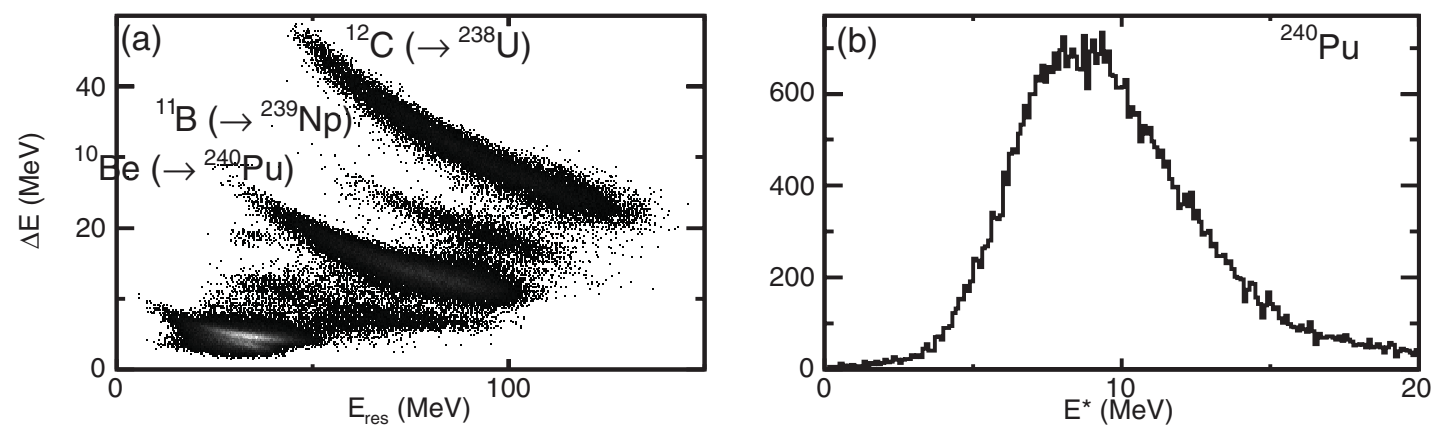

Figure 1. (a) Energy loss $(\Delta \mathrm{E})$ of the target-like recoil nuclei as a function of their residual energy $\left(\mathrm{E}_{\mathrm{res}}\right)$. (b) Excitation energy spectrum for ${ }^{240} \mathrm{Pu}$.

fission-fragment mass yields can be estimated [1]. Before reaching the saddle point, the compound nucleus may release a part of its excitation energy by evaporating particle. At the saddle point, the remain excitation energy defines the potential energy landscape which influences the fission-fragment distributions. Therefore, the study of the properties of these distributions may reveal some informations about the formation and the deexcitation of the compound nucleus.

The use of inverse kinematics to measure the atomic number of all the fission fragments has been developed at GSI in the 1990s [2]. Previously, the charge of the heavy fragments was rather difficult to determine. Using a spectrometer allows to measure of the same time the mass of the fragments.

\section{Experiments}

Two different experiments have been performed at GANIL, using ${ }^{238} \mathrm{U}$ beams at different energies impinging ${ }^{12} \mathrm{C}$ or ${ }^{9} \mathrm{Be}$ targets. Depending on the impact parameter, different actinides are produced by transfer or fusion reactions, giving access to a broad excitation-energy regime. The fission-fragment distributions of these actinides were investigated using two spectrometers, VAMOS [3] and LISE [4], for the low-energy and high-energy experiments, respectively.

\subsection{Specific features of the experiments}

In the first experiment, the beam impinged on a thin ${ }^{12} \mathrm{C}$ target with an energy of $6.1 \mathrm{~A} \mathrm{MeV}$. At this energy, transfer reactions represent about $10 \%$ of the total cross section [5]. The actinides produced by transfer reactions were tagged by the detection and the identification of the target recoils in a highly segmented annular silicon telescope, SPIDER [6], as shown in Figure 1a. The excitation energy of the actinides produced by transfer reactions was determined from the angle and the energy of the target recoil assuming a two-body kinematics. The excitation energy distribution was measured with a mean value around $9 \mathrm{MeV}$ (see Fig. 1b). In this work, the two-proton transfer channel, i.e. the production of ${ }^{240} \mathrm{Pu}$, is studied. A tiny beam-energy straggling in the thin target $\left(0.1 \mathrm{mg} / \mathrm{cm}^{2}\right)$ led to the production of compound nuclei in fusion reaction with $\mathrm{E}^{*}=45.4 \pm 0.3 \mathrm{MeV}$. In the case that fission occurred, one of the two fission fragments was identified with the VAMOS spectrometer.

In a second experiment at LISE, a ${ }^{238} \mathrm{U}$ beam of $24 \mathrm{~A} \mathrm{MeV}$ has been used. Thick carbon and beryllium targets $\left(15 \mathrm{mg} / \mathrm{cm}^{2}\right.$ for both targets) were used, which resulted in considerable beam-energy straggling. The centre of mass energy ranged from 164 to $208 \mathrm{MeV}$ with the beryllium target, and from 210 to $274 \mathrm{MeV}$ with the carbon target. 
Figure 2. Distribution of the fission-fragment atomic number $Z$ for both experiments (the full data set for ${ }^{250} \mathrm{Cf}$ is presented). The resolutions are $\Delta Z / Z=1.6 \%$ for the LISE experiment (a) and $1.7 \%$ for the VAMOS experiment (b).
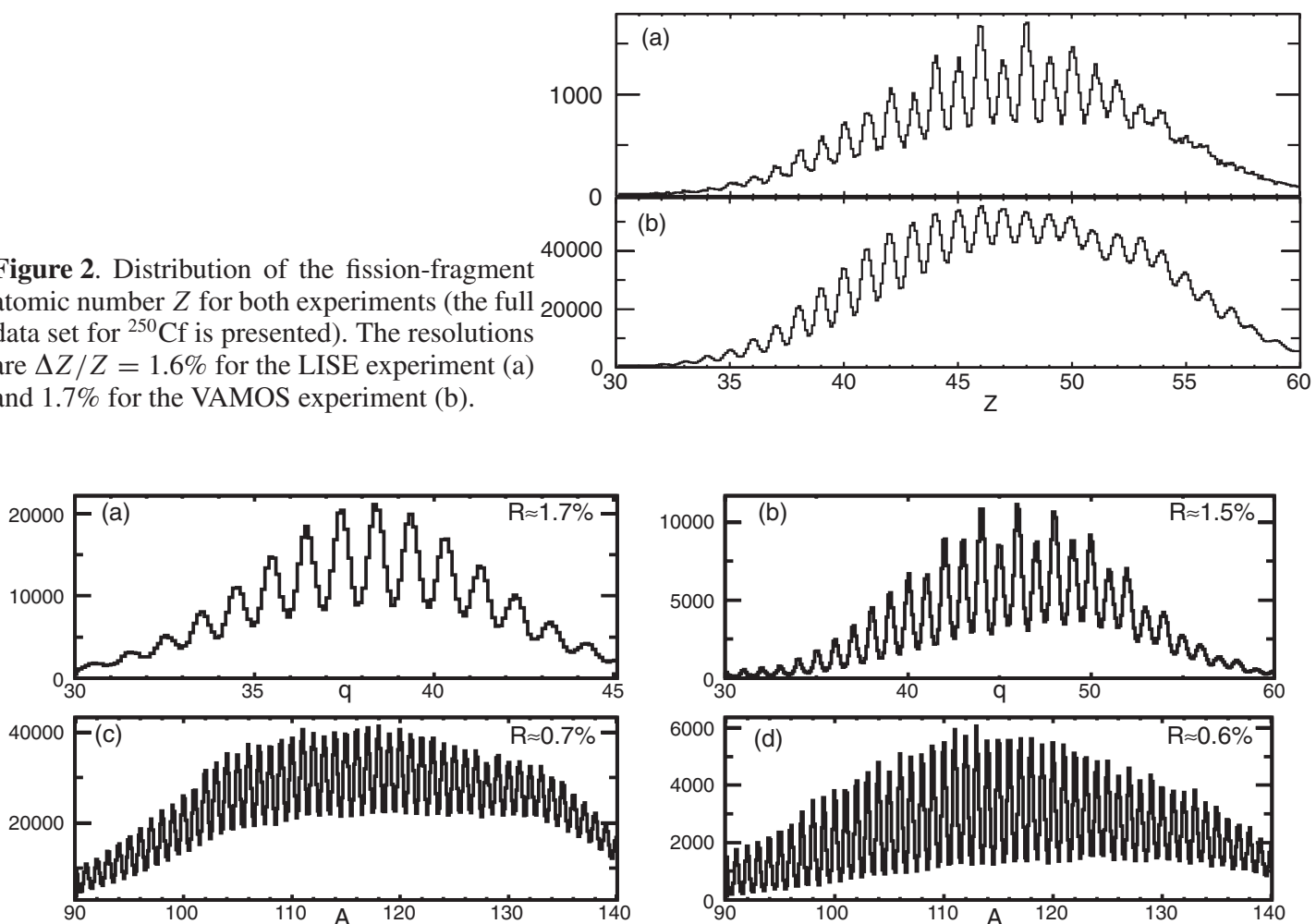

Figure 3. Ionic charge state $\mathrm{q}$ and mass A distributions measured from the fission of ${ }^{250} \mathrm{Cf}$ in VAMOS (a) and (c) and in LISE (b) and (d), respectively. Resolutions R are indicated in the figures.

In summary, different fissioning systems with 4 different excitation energies are investigated: ${ }^{240} \mathrm{Pu}$ with $\mathrm{E}^{*} \approx 9 \mathrm{MeV}$ (VAMOS), ${ }^{247} \mathrm{Cm}$ with $\mathrm{E}_{\mathrm{CM}} \approx 185 \mathrm{MeV}$ (LISE) and ${ }^{250} \mathrm{Cf}$ with $\mathrm{E}^{*}=45 \mathrm{MeV}$ $(\mathrm{VAMOS})$ and $\mathrm{E}_{\mathrm{CM}} \approx 240 \mathrm{MeV}$ (LISE).

\subsection{Identification of the fission fragments}

The fission-fragment identification was based in both experiments on the $\mathrm{B} \rho$-ToF- $\Delta \mathrm{E}-\mathrm{E}$ technique [7], where $\mathrm{B} \rho$ is the magnetic rigidity of the fragment, ToF its time of flight through the spectrometer and $\mathrm{E}$ its total kinetic energy. The identification of the atomic number $Z$ was obtained from energy-loss measurements. The atomic number distribution is shown in figure 2 for both experiments.

From the measurement of positions at the focal plane, the path and the radius of curvature, $\rho$, were determined [7]. The measurement of ToF gave the ion velocity v. The radius of curvature is related to the velocity by the relation $\mathrm{B} \rho=\mathrm{Av} / \mathrm{q}$, where $\mathrm{B}$ is the magnetic field applied to the dipoles of the spectrometers, $\mathrm{A}$ the mass of the ion, and $\mathrm{q}$ its ionic charge state. From $\mathrm{B} \rho$ and $\mathrm{v}$ measurements, the ratio $\mathrm{A} / \mathrm{q}$ was determined. The mass, $\mathrm{A}_{\mathrm{E}}$, can also be derived using the velocity and the energy. The ionic charge state is then determined as $\mathrm{q}=\frac{\mathrm{A}_{\mathrm{E}}}{\mathrm{A} / \mathrm{q}}$ (see Figs. 3a and $\mathrm{b}$ ). Finally, the mass A was obtained multiplying A/q by the integer value of q (see Figs. 3c and d). At VAMOS, a $\gamma$-ray detector located in the target region was used to validate the spectrometer identification [8] and at LISE the $\gamma$-ray detectors were installed at the focal plane and measured isomeric decay of fission fragments. 

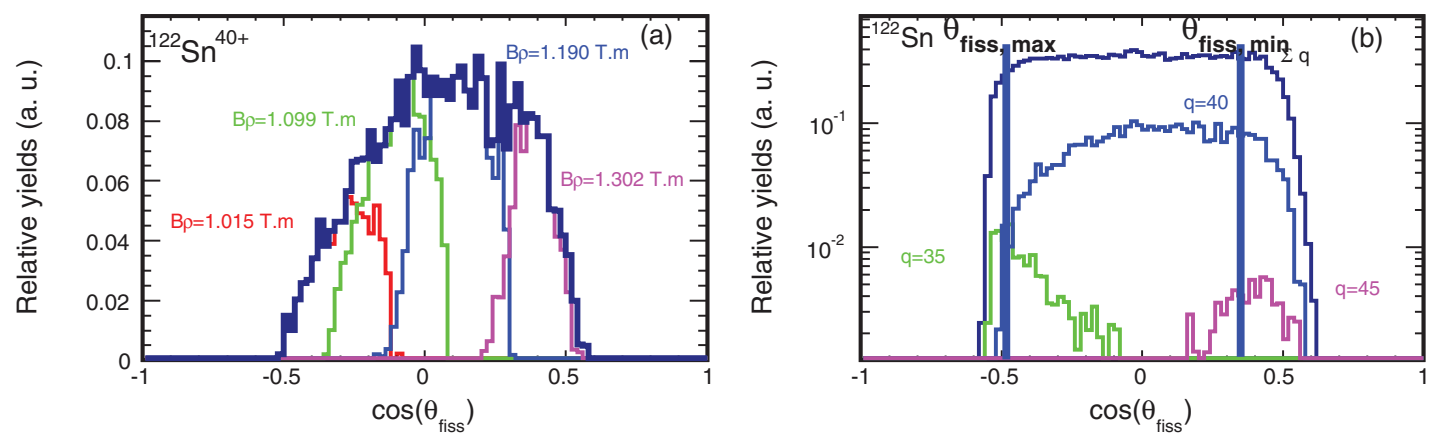

Figure 4. (a) Angular distribution of the ion ${ }^{122} \mathrm{Sn}^{40+}$. Angular distributions for different $\mathrm{B} \rho$ settings are plotted in colour. The total angular distribution was obtained taking the envelope of all the settings. (b) Angular distribution in the frame of the fissioning nucleus $\theta_{\text {fiss }}$ summing all ionic charge state contributions.

\section{Fission yields}

To determine isotopic fission yields $\mathrm{Y}(\mathrm{Z}, \mathrm{A})$, the first step is the reconstruction of the momentum distribution for each ionic charge state, using different $\mathrm{B} \rho$ settings of the spectrometer. The different runs are normalised to the beam intensity using the elastic scattering of the target in SPIDER in the case of the VAMOS experiment. A precision of $10 \%$ is obtained. In the case of the LISE experiment, the beam intensity was measured with a Faraday cup before and after each run.

\subsection{Normalisation and spectrometer acceptance correction}

Several $\mathrm{B} \rho$ values were needed to cover the angular distribution shown in figure 4(a). The distributions were corrected by the azimuthal angle $\varphi$ acceptance and normalised to the beam intensity. The acceptance on $\varphi$ depends on the value of the polar angle $\theta$ and the magnetic rigidity $\mathrm{B} \rho$ [7]. Thus, the correction was applied on an event per event basis, for each value of $\theta$ and $\mathrm{B} \rho$ of the fragments. Considering the envelope spectrum - shown as a black line in Figure $4 \mathrm{a}$ - a first estimation of the yield $\mathrm{Y}_{0}(\mathrm{Z}, \mathrm{A}, \mathrm{q})$ for each ion was obtained. The isotopic yield estimation is given by $\mathrm{Y}_{1}(\mathrm{Z}, \mathrm{A})=\sum_{q} \mathrm{Y}_{0}(\mathrm{Z}, \mathrm{A}, \mathrm{q})$. Finally, $\mathrm{Y}_{1}$ was corrected following $\theta$ using the relation $\mathrm{Y}(\mathrm{Z}, \mathrm{A})=\mathrm{Y}_{1}(\mathrm{Z}, \mathrm{A}) \frac{2}{\int_{\theta_{\text {fiss,min }}^{\theta}}^{\theta_{i n s} \sin \theta d \theta}}, \theta_{\text {fiss,min }}$ and $\theta_{\text {fiss, } \max }$ being the angles between which the events are fully transmitted, as illustrated in Figure $4 \mathrm{~b}$. A precise description of the reconstruction method is given in [9].

To correct the data of the LISE experiment from the spectrometer acceptance, a simulation based on the fission kinematics proposed by Wilkins was used [10]. We modelled also the charge state distribution to reproduce the measured one from the Schiwietz parametrisation [11]. Considering a square acceptance of $1^{\circ}$ in angle and $0.8 \%$ in magnetic rigidity, we determine from the simulation the correction factor as the ratio between the number of ion produced and the number of ions transmitted. The methods that we used to get the yields and to correct the transmission are presented in detail in [12].

\subsection{Results and discussion}

Following the methods described above, isotopic yields were obtained for the four different fissioning systems investigated, and for the complete fragment production. In the following, the main characteristics of the isotopic distributions, namely the neutron excess defined as the ratio of the mean neutron number value $\langle N\rangle$ over $Z$ and the neutron-number width $\sigma(N)$ are studied. 

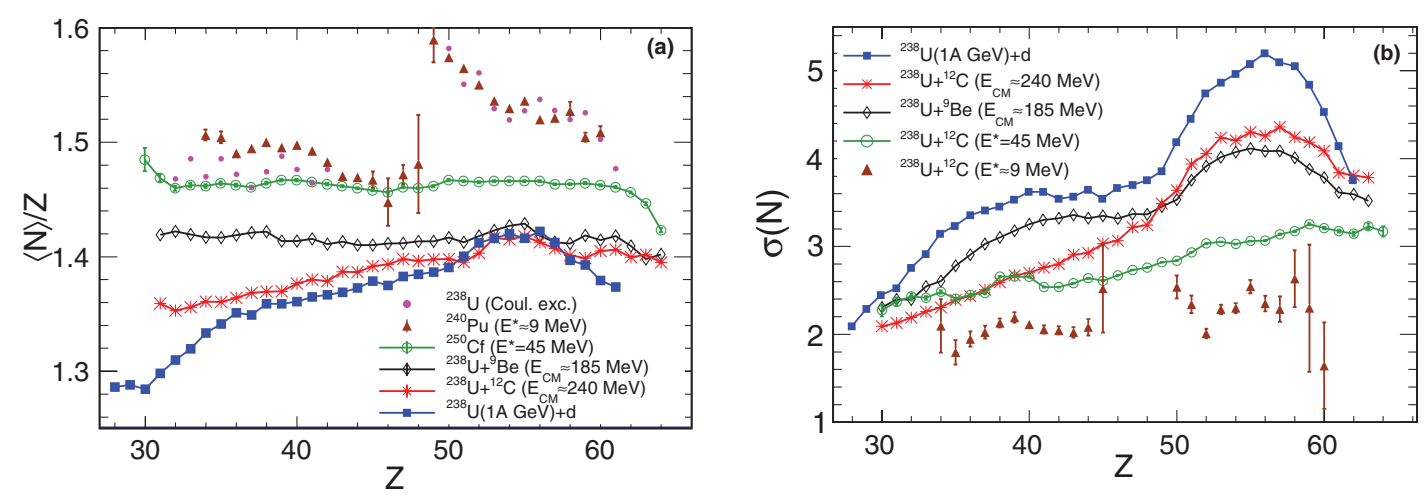

Figure 5. (a) Neutron richness $\langle N\rangle / Z$ as a function of the fission fragments atomic number $Z$ for different systems. (b) The width of the neutron-number distribution $\sigma(N)$ as a function of the fission fragments atomic number $Z$ for different systems.

Figure 5a shows the evolution of the mean neutron excess, $\langle N\rangle / Z$, with the fission-fragment atomic number $Z$ for the different fissioning systems and different excitation energies. At low excitation energy (triangles), light fragments exhibit lower $\langle N\rangle / Z$ values than heavy ones. This can be understood as the result of the minimisation of the total potential energy of the emerging fragments: an excess of neutrons in the heavy fragments lowers the influence of the Coulomb energy contribution and symmetry energy in the total potential energy [10]. This trend is enhanced by shell effects around $Z=50$. For the fission of ${ }^{250} \mathrm{Cf}$ produced at $45 \mathrm{MeV}$ (circles), $\langle N\rangle / Z$ gives a constant value of 1.47 , which is consistent with the N/Z ratio of ${ }^{242} \mathrm{Cf}$. This would mean that 8 neutrons in total would be evaporated by the compound nucleus and the fission fragments, independently of the mass of the fission fragment. At higher centre of mass energy (greater than $160 \mathrm{MeV}$ ) (diamonds and asterisks), the $\langle N\rangle / Z$ ratio decreases as the centre of mass energy increases, indicating more evaporated neutrons. A hump is formed around $Z \approx 54$. This can be understood as the effect of different entrance channels with different excitation energies. At high bombarding energy, pre-equilibrium particles emission may occur before the formation of the compound nucleus and consequently, the different compound nuclei are produced over a range of $\mathrm{A}$ and $\mathrm{E}^{*}$. Fission at a low excitation energy induces the hump around $Z \approx 54$ from shell effects which stabilise the mass and atomic-number distributions of heavy fission fragments [13]. This trend is confirmed by data from spallation-fission reactions performed at GSI [14], for ${ }^{238} \mathrm{U}$ at $1 \mathrm{~A} \mathrm{GeV}$ impinging on a deuterium target (squares). Figure $5 \mathrm{~b}$ shows the width of the isotopic distribution $\sigma(N)$ as a function of the fissionfragment atomic number for the same fissioning systems. From a statistical description of the fission process, $\sigma(N)$ is expected to increase with the temperature T and with the mass $\mathrm{A}$ [15], in agreement with the present data. The large energy straggling existing in the highest energy reactions certainly contributes to enlarge the distributions. Likewise for $\langle N\rangle / Z$, a large increase of the width is observed around $\mathrm{Z} \approx 54$ at high bombarding energy, which most likely reflects the presence of different entrance channels with different excitation energies.

The fragments from the fission of ${ }^{250} \mathrm{Cf}$ produced at $\mathrm{E}^{*} \approx 45 \mathrm{MeV}$ show a different behaviour, with constant $\langle N\rangle / Z$ and a regular increase in $\sigma(N)$. In this reaction, shell effects are expected to be weak and entrance-channel effects to be limited. Thus, a liquid-drop behaviour is expected, i.e. $\langle N\rangle / Z$ at scission is expected to increase steadily with $\mathrm{Z}$ [16]. The observed constant value of $\langle N\rangle / Z$ with $Z$ suggests that heavy fission fragments evaporate more neutrons than light ones and compensate exactly the neutron excess of fragments at scission. The absence of a shell-gap influence, supported by the lack of hump in the evolution of $\sigma(N)$ with $\mathrm{Z}$, puts forward that the compound nucleus has no time to 
evaporate neutrons before reaching the saddle deformation, i.e. first-chance fission is highly probable, as was already suggested in the study of similar fissioning systems at similar excitation energy [17].

\section{Conclusion}

The technique of the inverse kinematics combined with a spectrometer is a powerful tool to investigate fission-fragment distributions. The complete isotopic fission-fragment yields are measured for different fissioning systems at different excitation energies. The production of neutron-rich fission fragments reveals to be a complex process where the influence of the entrance channel effects is decisive. The neutron-excess degree of freedom shows to be very powerful in gaining information about time scales and dynamics of low-energy nuclear reactions. In particular, the present results indicate that at moderate excitation energy the compound nucleus reaches the saddle deformation before any significant cooling by neutron evaporation, and that the fission fragments release the remaining excitation energy.

\section{References}

[1] Goutte H. et al., Phys. Rev. C 71 (2005) 024316

[2] Bernas M. Nucl. Phys. A 616 (1997) 352-362

[3] Savajols H. et al., Nucl. Instr. and Meth. B 204 (2003) 146-153

[4] Anne R. et al., Nucl. Instr. and Meth. A 257 (1987) 215-232

[5] Biswas D. C. et al., Phys. Rev. C 56 (1997) 1926-1935

[6] Derkx X. et al., EPJ Web of Conferences, 2010, 2, 07001 CNR09 - Second International Workshop on Compound Nuclear Reactions and Related Topics - Bordeaux, France

[7] Pullanhiotan S. et al., Nucl. Instr. and Meth. A 593 (2008) 343-352

[8] Shrivastava A. et al., Phys. Rev. C 80 (2009) 051305

[9] Caamaño M. et al., arXiv:1304.2647 [nucl-ex]

[10] Wilkins B. D. et al., Phys. Rev. C 14 (1976) 1832

[11] Schiwietz, G. and Grande, P. Nucl. Instr. and Meth. B 175-177 (2001) 125-131

[12] Delaune O. et al., arXiv:1302.1981 [nucl-ex]

[13] Böckstiegel, C. et al., Nucl. Phys. A 802 (2008) 12-25

[14] Pereira J. et al., Phys. Rev. C 75 (2007) 014602

[15] Yuri T. S. Oganessian and Yuri A. Lazarev, Treatise on Heavy-Ion Science 4, Plenum Press New York, 1985

[16] Berlanger M. et al. Z. Phys. A 291 (1979) 133-143

[17] Rubehn Th. et al., Phys. Rev. C 54 (1996) 3062-3067 